THE Astrophysical JourNAL, 390:679-686, 1992 May 10

(C) 1992. The American Astronomical Society. All rights reserved. Printed in U.S.A.

\title{
MAGNETIC RECONNECTION IN INCOMPRESSIBLE FLUIDS
}

\author{
Edward E. DeLuca \\ University of Hawaii, Institute for Astronomy, 2680 Woodlawn Drive, Honololu, HI 96822 \\ AND \\ IAN J. D. CRAIG \\ University of Waikato, Department of Mathematics and Statistics, Private Bay, Hamilton, New Zealand \\ Received 1991 July 9 ; accepted 1991 November 12
}

\begin{abstract}
We investigate the dynamical relaxation of a disturbed $X$-type magnetic neutral point in a periodic geometry, with an ignorable coordinate, for an incompressible fluid. We find that the properties of the current sheet cannot be understood in terms of steady state reconnection theory or more recent linear dynamical solutions. Accordingly we present a new scaling law for magnetic reconnection consistent with fast energy dissipation, i.e., the dissipation rate at current maximum is approximately independent of magnetic diffusivity $(\eta)$. The flux annihilation rate, however, scales as $\eta^{1 / 4}$, faster than the Sweet-Parker rate of $\eta^{1 / 2}$ but asymptotically much slower than the dissipation rate. These results suggest a flux pile-up regime in which the bulk of the free magnetic energy is released as heat rather than as kinetic energy of mass motion.
\end{abstract}

The implications of our results for reconnection in the solar atmosphere and interior are discussed.

Subject headings: MHD - Sun: flares - Sun: magnetic fields

\section{INTRODUCTION}

Magnetic fields in the Sun are continually changing on time scales that range from seconds to tens of years. Topological changes in the magnetic field are needed to release energy in solar flares (100 s time scales) and to change the orientation of the Sun's toroidal field (11 yr time scales). The only way the topology of a magnetic field can change is through the effect of finite resistivity. The time scales for such changes scale as $t_{\eta} \sim$ $L^{2} / \eta_{*}$, where $\eta_{*}=c^{2} / 4 \pi \sigma$ is the magnetic diffusivity of the fluid and $L$ is a characteristic length scale of the magnetic field. Since the solar plasma has a high conductivity $\sigma \approx 10^{7} T^{3 / 2}$ e.s.u. (Spitzer 1956; Parker 1979) the characteristic scale over which the magnetic field changes must be very small for solar flares $\left(T \approx 10^{6} \mathrm{~K}\right) l=30 \mathrm{~m}$ but comparatively large $\left(T \approx 10^{6} \mathrm{~K}\right)$ $l=50,000 \mathrm{~km}$ for the toroidal field of the solar cycle.

How strong magnetic field gradients are formed in the Sun depends, in part, on the form of the magnetic field. If the field is fibril, then strong gradients may be formed when oppositely directed fibrils are brought together. If the field is diffuse, shearing motions associated with turbulent eddies can produce strong field gradients. While it is well recognized that the surface magnetic field is fibril, models of the magnetic field generation within the convection zone often assume a diffuse magnetic field (DeLuca \& Gilman 1991; Stix 1989). It is assumed in these models that the turbulent diffusion can efficiently dissipate the magnetic field on time scales comparable to the solar cycle. Recent work by Vainshtein and collaborators (Vainshtein \& Rosner 1991; Cattaneo \& Vainshtein 1991; Vainshtein, Parker, \& Rosner 1991) has cast doubts on the efficiency of turbulent diffusion.

The present paper is motivated by the possibility that fields in the convection zone are fibril in nature and that magnetic reconnection of oppositely directed fields is responsible for the changes seen in the toroidal magnetic field. For magnetic reconnection problems the major distinction between the solar atmosphere and interior is the compressibility of the fluid. To model conditions in the solar convection zone we assume that the fluid is incompressible. An independent compressible coronal calculation has recently been performed by Craig \& McClymont (1992). The fact that the coronal calculation shows substantially the same development as our incompressible current sheet suggests that our findings may apply within a wider context than the solar convection zone. Indeed, steady state flare reconnection theory traditionally assumes an incompressible fluid (e.g., Parker 1963; Petschek 1964).

In the following section we present a new scaling for the nonlinear evolution of a current sheet in our dynamical calculations and contrast our scaling laws with both the steady state models (Forbes \& Priest 1987; Petschek 1964) and the recent linear dynamical solutions of Craig \& McClymont (1991). In $\S 3$ the model equations and numerical method are presented. The results are discussed in $\S 4$. The implications of our results for magnetic fields in the solar atmosphere and interior are presented in $\S 5$.

\section{SCALING LAWS}

When considering the reconnection of magnetic fields, the key question becomes the dependence of the rate of reconnection on the magnetic Reynolds number, $R_{m}=L V / \eta_{*}$, where $L$ is a characteristic length scale and $V$ a characteristic velocity. In the Sun the magnetic diffusivity is so small and the length scales are so large that only a very weak dependence of the reconnection rate on $R_{m}$ is tolerable. We will define the reconnection rate to be fast if the dependence on $R_{m}$ is weaker than any power law. (Note: we will often use $\eta \equiv R_{m}^{-1}$ the dimensionless magnetic diffusivity, in the $\operatorname{Sun} \eta \sim 10^{-12}$ ). The rate of reconnection is known to depend on the geometry of the neutral point and the boundary conditions on the velocity and magnetic fields (see Forbes \& Priest 1987). In our calculations we consider the spontaneous reconnection of a disturbed $\mathrm{X}$-type neutral point in a periodic geometry. The computation is entirely dynamic allowing for simple and unambiguously specified boundary and initial conditions - and thus eluding many of the interpretive difficulties associated with driven 
TABLE 1

SCALING Properties of Different MODELS

\begin{tabular}{|c|c|c|c|c|c|c|}
\hline \multirow[b]{2}{*}{ MODEL } & \multirow[b]{2}{*}{ CoMments } & \multirow[b]{2}{*}{$l$} & \multirow[b]{2}{*}{$L$} & \multirow[b]{2}{*}{$J_{\max }$} & \multicolumn{2}{|c|}{$\sim\left(\eta J_{\max }^{2} l L\right) \sim-\left(\eta J_{\max }\right)$} \\
\hline & & & & & $W_{\eta}$ & $\dot{\psi}$ \\
\hline Sweet-Parker . & Order of magnitude & $\eta^{1 / 2}$ & $\eta^{0}$ & $\eta^{-1 / 2}$ & $\eta^{1 / 2}$ & $\eta^{1 / 2}$ \\
\hline Petschek ..................... & Semi-analytic & $\eta$ & $\eta$ & $\eta^{-1}$ & $\eta$ & $\ln (\eta)$ \\
\hline Craig \& McClymont $1991 \ldots \ldots \ldots \ldots \ldots \ldots \ldots$ & Linear exact & $\eta^{1 / 2}$ & $\eta^{1 / 2}$ & $\eta^{-1}$ & $\ln (\eta)^{2}$ & $\ln (\eta)$ \\
\hline Biskamp \& Welter ............................ & Empirical & $\eta^{2 / 3}$ & $\eta^{0}$ & $\eta^{-1}$ & $\eta^{-1 / 3}$ & $\eta^{\circ}$ \\
\hline Flux pile-up & Empirical & $\eta^{1 / 2}$ & $\eta^{0}$ & $\eta^{-3 / 4}$ & $\eta^{0}$ & $\eta^{1 / 4}$ \\
\hline
\end{tabular}

a Both the Craig \& McClymont 1992 coronal calculation and our incompressible calculation are well represented by the flux pile-up model.

steady state reconnection studies as discussed by Forbes \& Priest (1987). However, the interpretation of the solution may require more care since steady state measures of "fastreconnection" no longer apply.

In the steady problem the rate of reconnection is measured by the inflow speed of the advected "external" magnetic field into the diffusion layer. The earliest result is the order of magnitude calculation of Sweet (1958) and Parker $(1957,1963)$ in which the reconnection rate scales as $\eta^{1 / 2}$. This rate is too slow for solar flares but Petschek (1964) showed that a "fast," $|\ln (\eta)|$, rate could be achieved, for steady state driven flow, by modifying the flow topology in the advection region. More recent steady state modeling has confirmed that fast magnetic merging is possible for a far wider class of flow topologies (Forbes \& Priest 1987). That fast reconnection can be achieved dynamically for prescribed initial conditions is shown by the exact linear calculations of Craig \& McClymont (1991). Their analysis breaks down, however, for finite amplitude energy release (Craig \& McClymont 1992).

In a dynamic calculation, the steady state reconnection rate has little meaning, and it is far more instructive to analyze the properties of the current sheet, in particular the dependence of the length, breadth, and current density with resistivity. In the Sweet-Parker mechanism the length of the sheet is invariant (fixed by the geometry), the width scales as $\eta^{1 / 2}$ and the current density has an $\eta^{-1 / 2}$ dependence. Table 1 summarizes the dependencies of the current sheet based on a selection of reconnection studies. It should be remarked that an important aspect of the Petschek calculation is the fixed aspect ratio of the current sheet with resistivity-a property also shared by the fast mechanism of Craig \& McClymont (1991). The steady state numerical simulations of Biskamp (1986) are contrary to this behavior and are more in keeping with the scalings of the Sweet-Parker mode (although, see the critique of Forbes \& Priest 1987).

The table also indicates the scaling of "flux pile-up" solutions. Although pile-up solutions can be difficult to interpret in terms of steady state theory (Forbes \& Priest 1987), they appear to be a common feature of dynamic simulations. A rationale for the pile-up scaling is as follows:

Consider disturbing a force free equilibrium by a perturbation; the perturbation can be thought of as consisting of two parts: a part that has distorted the equilibrium without changing the topology and a part that has changed the topology of the equilibrium. The former perturbations can be removed by fluid motions alone, while the latter require reconnection to be removed. If all dissipation mechanisms are absent then the perturbation energy must ultimately appear as the magnetic and kinetic energy associated with the formation of singular current structures. If viscous dissipation is allowed, but no ohmic dissipation, the kinetic energy will be dissipated, and we will be left with the perturbation magnetic energy contained in singular current sheets. Hence for a fixed perturbation amplitude and small enough resistivity the magnetic energy associated with the sheet formation should be independent of the resistivity. Craig \& Watson (1991) give an exact analytic description of the formation of the current singularity for the case of small displacements about simple two-dimensional $\mathrm{X}$-point equilibria.

In the present analysis the $\mathrm{X}$-point is three-dimensional but possesses an ignorable $z$-coordinate. Accordingly, the magnetic energy in the current sheets is proportional to $B_{s}^{2} a_{s}$, where $B_{s}$ is the characteristic magnetic field strength in the sheet and $a_{s}$ is the area of the sheet. Now $a_{s} \sim r^{2}$ or $l^{2}$ if the current "sheet" is cylindrical (as is the case for the linear Craig \& McClymont 1991 model), and $a_{s} \sim l L$ if the current sheet is long and thin. (We use $l$ to denote the width of the current sheet and $L$ to denote the length.) The morphology of the current singularity is therefore a critical factor in the reconnection problem.

Consider first the case where the current structure is cylindrical - the case of small amplitude disturbances. Then the implosive collapse toward singularity is governed by cylindrical wave equation $\psi_{t t}=r^{2} \nabla^{2} \psi$ (Craig \& Watson 1991). (The Alfvén speed is proportional to $r$ if we assume that the field varies linearly with the distance from the neutral point.) It follows on equating the diffusion $(\eta / r)$ and advection $(r)$ speeds that the area of the sheet must scale as $\eta$. The invariance of the magnetic energy in the sheet then leads to the Craig \& McClymont (1991) scaling summarized in Table $1\left(r^{2}=\right.$ $\left.l^{2} \sim \eta, B_{s}^{2} r^{2} \sim \eta^{0} \Rightarrow B_{s} \sim \eta^{-1 / 2} ; J_{\max } \sim B_{s} / l \sim \eta^{-1}\right)$. For this scaling the total current in the sheet $I_{s}=J_{\max } a_{s}$ is independent of the resistivity.

For larger amplitude disturbances, however, the cylindrical structure is lost as the background field becomes overwhelmed by the perturbation field. The sheet becomes quasi-onedimensional - the length of the sheet being determined by the local cancellation of the background field at some stage during the advective implosion. What follows is a highly nonlinear quasi-one-dimensional implosive collapse toward long thin singular current sheet. In the simplest approximation we replace the cylindrical wave equation by its one-dimensional counterpart $\psi_{t t}=x^{2} \nabla^{2} \psi$. (The magnetic field is assumed to vary linearly with distance from the neutral line.) Matching the diffusive and advective speeds again gives $x=l \sim \eta^{1 / 2}$ but now the length of the current sheet $L \sim \eta^{0}$. Combining these scalings with the assumption that the total magnetic energy in the 
sheet is constant give the flux pile-up scaling summarized in Table $1\left(B_{s}^{2} l L \sim \eta^{0} \Rightarrow B_{s} \sim \eta^{-1 / 4} ; J_{\max } \sim B_{s} / l \sim \eta^{-3 / 4}\right)$. While the width in this model scales as $\eta^{1 / 2}$ the total current in the layer builds up as $J_{\max } l L \sim \eta^{-1 / 4}$. It is this built-up current/ flux over the diffusive layer that allows the possibility of fast reconnection.

For time-dependent problems two definitions of the reconnection rate will be employed: one measure is the rate of flux annihilation at the neutral point given by $\partial \psi / \partial t \sim-\eta\left|J_{\max }\right|$ where $\psi$ is the flux function and $J_{\max }$ is the maximum current in the sheet. An alternative is the energy dissipation rate, $W_{\eta} \equiv$ $\eta \int J^{2} d s$, determining the conversion of free magnetic energy to heat in the current sheet. These measures are discussed in the Biskamp studies (Biskamp \& Welter 1980; Biskamp 1986) and summarized in Table 1 for the various reconnection models. The Biskamp \& Welter scaling for the energy dissipation rate must break down as $\eta \rightarrow 0$; they argue that below a critical value of $\eta$ the reconnection rate will approach the SweetParker rate.

For brevity a mechanism is described as "fast" if either $\partial \psi / \partial t$ or $W_{\eta}$ are independent of any power or $\eta$. In fact only the dynamic model of Craig \& McClymont (1991) is fast according to both measures; yet it is consistent with the Petschek mechanism in that flux annihilation dominates ohmic dissipation in the limit of small $\eta$. More generally, our diagnostics determine whether the excess magnetic energy is converted mainly to heat or to kinetic energy of mass motion. In what follows we approximate the energy dissipation rate as $W_{\eta} \sim \eta J_{\max }^{2} L l$.

Recently, Biskamp \& Welter (1989) have examined the scaling properties of decaying, two-dimensional, MHD turbulence. They use very high resolution $\left(1024^{2}\right)$ simulations to resolve the dissipation scales of the high Reynolds number calculations. Figure 10 of that paper shows the development of long thin current sheets after just 1.5-2.0 Alfvén transit times. These current sheets are clearly limited in length by the geometry of the box. The current sheets in this calculation are unstable to tearing modes and quickly generated fine-scaled structures within the sheet. Biskamp \& Welter (1989) argue that after the current sheets become turbulent the dissipation rate becomes independent of $\eta$. We are attempting to show that the necessary conditions for fast reconnection occur in a laminar current sheet around the time of current maximum.

\section{MODEL EQUATIONS AND NUMERICAL METHOD}

Our model equations are the viscous, resistive MHD equations for an incompressible fluid. The full equations are

$$
\begin{gathered}
\rho \frac{\partial \boldsymbol{u}}{\partial t}+\rho(\boldsymbol{\omega} \times \boldsymbol{u})=-\nabla P+\frac{1}{4 \pi} \boldsymbol{J} \times \boldsymbol{B}+\rho v_{*} \nabla^{2} \boldsymbol{u} \\
\frac{\partial \boldsymbol{B}}{\partial t}+(\boldsymbol{u} \cdot \nabla) \boldsymbol{B}=(\boldsymbol{B} \cdot \nabla) \boldsymbol{u}+\eta_{*} \nabla^{2} \boldsymbol{B}, \\
\nabla \cdot \boldsymbol{u}=0 \\
\nabla \cdot \boldsymbol{B}=0 .
\end{gathered}
$$

Here $\boldsymbol{u}$ and $\boldsymbol{B}$ are the velocity and magnetic fields; $P=P_{\text {gas }}$ $+\frac{1}{2} \rho u^{2}$ is the pressure; $\boldsymbol{J}=\nabla \times B$ is the current; $\rho$ is the density (assumed constant); $v_{*}$ is the kinematic viscosity, and $\eta_{*}$ is the magnetic diffusivity.

The dimensionless equations are found by defining a characteristic speed, magnetic field strength and length scale for the problem. Because the velocity fields in our problem result from the Lorentz force, both the velocity and the magnetic field are scaled by the Alfvén speed, $V_{\mathrm{A}} \equiv\left[B^{2} /(4 \pi \rho)\right]^{1 / 2}$. The characteristic length scale is $\chi$. (We will show below that the length of the current sheet $L$ is limited by $\chi$ the limit of small diffusivity.) The characteristic time scale is the Alfvén crossing time $t_{A}=\chi / V_{\mathrm{A}}$ (our computational box will range from 0 to $2 \pi \chi$ so the Alfvén crossing time for the entire box will be $\left.t \simeq 6.3 t_{\mathrm{A}}\right)$. The pressure scales as $V_{\mathrm{A}}^{2}$, the vorticity scales as $V_{\mathrm{A}} / \chi$, and the current scales as $V_{\mathrm{A}} / \chi$. Two-dimensionless numbers define the parameter space for our problem: the Reynolds number $R_{e} \equiv L V_{\mathrm{A}} / v_{*}$ and the Lundquist number $L_{u}=\eta^{-1} \equiv L V_{\mathrm{A}} / \eta_{*}$ (or magnetic Reynolds number). The dimensionless momentum equation is

$$
\frac{\partial u}{\partial t}=-\omega \times u-\nabla P+J \times B+R_{e}^{-1} \nabla^{2} u
$$

The assumption that we have an ignorable coordinate $(\partial f /$ $\partial z=0 \forall f$ ), allows us to satisfy equation (4) by defining a flux function $\psi$. With $\boldsymbol{B}=\left(\psi_{y},-\psi_{x}, b\right)$ and $\boldsymbol{u}=(u, v, w)$ the dimensionless induction equation becomes

$$
\frac{\partial \psi}{\partial t}=-(\boldsymbol{u} \cdot \nabla) \psi+\eta \nabla^{2} \psi
$$

for $\psi$ and

$$
\frac{\partial b}{\partial t}=-(\boldsymbol{u} \cdot \nabla) b+\left(\psi_{y} w_{x}-\psi_{x} w_{y}\right)+\eta \nabla^{2} b,
$$

for $b$; the notation $\psi_{x} \equiv \partial x$ is employed. We choose to satisfy the incompressibility constraint, equation (4), by solving

$$
\nabla^{2} P=\nabla \cdot(J \times B)+\nabla \cdot(\omega \times u) .
$$

Our numerical technique involves the use of a pseudospectral collocation method for the spatial dependencies, and a combination of implicit and explicit time stepping routines. The diffusion terms are treated implicitly by a second-order Crank-Nicolson method and the nonlinear terms explicitly via a second-order Adams-Bashforth method (see Canuto et al. 1988 for a discussion of these methods).

\section{RESULTS}

Table 2 summarizes some of the properties of the simulations we discuss below. We begin with a description of the large-scale features of the solution and then discuss the scaling properties of the current sheets.

\subsection{Morphology of the Solutions}

It is important to emphasize that the possibility of fast reconnection in a closed topology, incompressible plasma demands initial conditions that are not too simple. For example, disturbances of a single $X$-type neutral point decay only on the static diffusion time scale. The reason is clear: if the initial condition has too much symmetry the Lorentz force can always be balanced by a pressure gradient force and there will be no flow. Thus fast reconnection in an incompressible fluid requires either inflow and outflow boundary conditions on the side walls (a driven problem) or else a closed circulation.

In our formulation an initial, periodic magnetic field is specified in a zero initial velocity field. The initial field consists of an equilibrium field $\boldsymbol{B}_{\mathrm{eq}}=\left(\psi_{y},-\psi_{x}, \sqrt{2} \psi\right)$, where $\psi=\sin x \sin y$, and a perturbation $\boldsymbol{B}_{\text {pert }}=\left(\psi_{y}^{\prime},-\psi_{x}^{\prime}, 0\right)$, where $\psi^{\prime}=\cos ^{2} y$. The initial magnetic field configuration is shown in Figure 1 (Plate 12). The arrows indicate the amplitude and direction of 


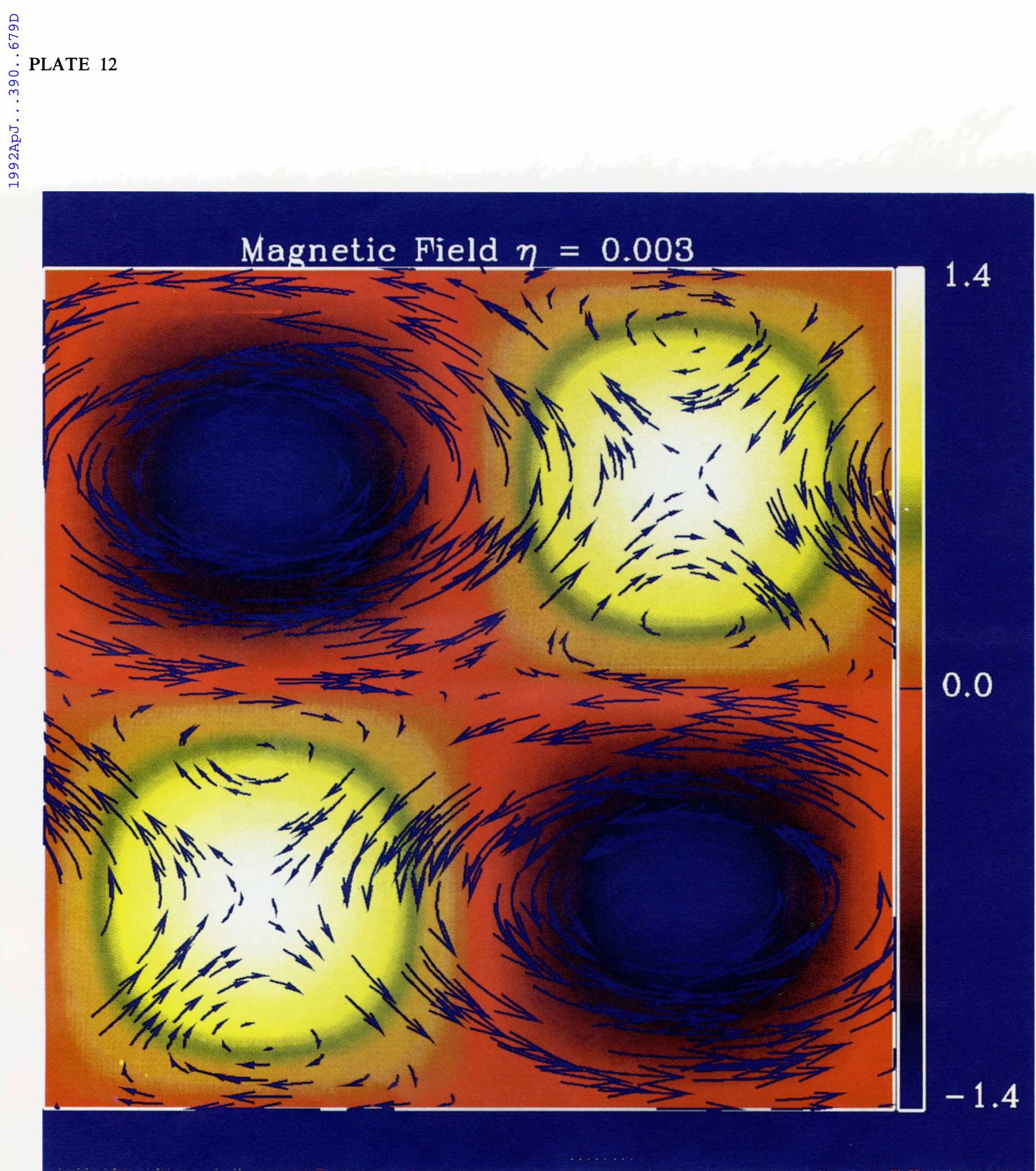

FIG. 1.- The initial magnetic field. The horizontal field is represented by arrows, the vertical field by colors; yellow-white positive (out of the page), red about zero, blue-black negative.

Deluca \& Craig (see 390, 681) 
TABLE 2

SUMMARY OF SOLUTIONS

\begin{tabular}{|c|c|c|c|c|c|c|c|c|c|c|}
\hline$\eta$ & $L_{u}$ & $N$ & $l$ & $L$ & $J_{\max }$ & $B_{x}$ & $W_{\eta}$ & $I$ & ke & $\Delta m e$ \\
\hline 0.03 & 33 & 48 & 0.22 & 1.84 & 1.94 & 0.29 & 0.014 & 0.40 & 3.40 & 0.68 \\
\hline $0.02 \ldots \ldots \ldots \ldots$ & 50 & 64 & 0.16 & 1.76 & 3.25 & 0.33 & 0.028 & 0.63 & 3.58 & 1.97 \\
\hline $0.01 \ldots \ldots \ldots \ldots$ & 100 & 64 & 0.11 & 1.76 & 6.75 & 0.49 & 0.056 & 1.07 & 3.78 & 3.71 \\
\hline $0.005 \ldots \ldots \ldots \ldots$ & 200 & 128 & 0.07 & 1.96 & 12.77 & 0.67 & 0.070 & 1.98 & 3.89 & 4.69 \\
\hline $0.003 \ldots \ldots \ldots \ldots$ & 333 & 128 & 0.05 & 2.06 & 18.77 & 0.80 & 0.081 & 2.41 & 3.94 & 5.10 \\
\hline $0.002 \ldots \ldots \ldots \ldots$ & 500 & 128 & 0.04 & 2.16 & 25.12 & 0.85 & 0.085 & 2.65 & 3.96 & 5.35 \\
\hline $0.001 \ldots \ldots \ldots \ldots$ & 1000 & 192 & 0.03 & 2.36 & 38.92 & 0.98 & 0.079 & 3.41 & 3.99 & 5.61 \\
\hline $0.0005 \ldots \ldots \ldots$ & 2000 & 384 & 0.02 & 2.31 & 60.57 & 1.11 & 0.068 & 3.92 & 4.00 & 5.73 \\
\hline
\end{tabular}

Notes.-The Reynolds number $R_{e}=10000$ for all the solutions. $N$ is the number of collocation points used in each direction. $B_{x}$ is the strength of the magnetic field at the edge of the current sheet. $I=\sum J \delta x \delta y$ over the current sheet. All the quantities are measured at the time of current maximum except the kinetic energy and magnetic energy which are measured at the time of kinetic energy maximum.

the horizontal magnetic field, and the colors show the amplitude of the perpendicular magnetic field. There are three $X$-type neutral points (X's) in the figure, two nearly perpendicular X's in the lower left and upper right-hand side of the figure and an oblique $X$ in the middle of the figure. In what follows we shall regard the lower left and upper right-hand X's as the primary X's-in fact these are created when the perturbation is superposed on the equilibrium field.

It is important to remember that the perturbation changes the intrinsic topology of the equilibrium configuration. This means that the equilibrium can only be regained through reconnection, and dissipation. The need for topological change can be demonstrated by running simulations with no resistivity, $\eta=0$, but a large viscosity. The final state is then a singular force free configuration with current sheets (of resolution dependent amplitude) overlaying the primary X's. For the initial conditions described above, $59 \%$ of the perturbed energy can be dissipated by the fluid motions alone. The remaining energy is stored in the singular structures of the final configuration. The magnetic energy that needs to be dissipated in the current sheets (when $\psi$ is finite), is $14 \%$ of the total initial energy.

A salient feature of Figure 1 is the six O-type neutral points (O's). The primary O's, in the upper left and lower right-hand side, are topologically the same as O's in the equilibrium field. They are distorted from their circular shape by the imposed perturbation, but no reconnection is needed to restore them to their equilibrium shape. The four weak O's above and below the each of primary X's are the result of topological changes caused by the perturbation. During the relaxation process the initial magnetic field tends to relax back to the equilibrium field with O-type neutral points in place of the primary X's. The strong current sheets are formed by the coalescence of the weak O's.

Figure 2 (Plate 13) shows the horizontal velocity field and vertical current at time $t=0.25$. The coalescence of the weak O-type neutral points is manifested by the emergence of a vigorous circulation pattern. Much of this initial flow results from the primary O's tending to relax to their circular shape. Notice that the outflow from one of the primary X's forms the inflow of the other. As already mentioned, the formation of strong circulation patterns that connect the two current sheets is an important feature of our solutions - it allows the possibility of fast reconnection. There is a weaker circulation through the center $\mathrm{X}$, which will also form a current sheet.
The evolution of the velocity, magnetic field, and vertical current are shown for $\eta=0.03$ in Figures $3 a$ and $3 b$ (Plates 14-15). The color bar defines the amplitudes of the vertical magnetic field and the vertical current in the two figures, respectively. In the first frame of Figure $3 a$ we see that after 1 time unit the magnetic field has changed only slightly from the initial configuration. In the second frame oppositely directed field lines are clearly visible where the primary X's were located. The weak O's are moving toward the current sheet and become wider. In the third frame the weak O's have become squared off. The current sheet has reached its maximum length and is starting to contract by this point. Finally after 4 time units the O-type points have almost coalesced, and the current is nearly completely dissipated.

In Figure $3 b$ we see that the centers of the primary O's are becoming force free as manifested by the weak velocity field. As the primary O's relax to their force free state, the circulation is excluded from these regions. In the first frame the vertical current is essentially unchanged from its initial configuration (note that the color scale in Fig. $3 b$ is different from that in 2). The circulation still has nearly square cells with a small "figure eight" around the central X. These cells become constricted as the system evolves $(t=2)$, countercells are formed $(t=3)$, and finally the flow is reversed $(t=4)$.

The flows are driven by the Lorentz force subject to the constraint that $\boldsymbol{\nabla} \cdot \boldsymbol{u}=0$. Initially the flow is dominated by the relaxation of the primary O's and X's. As the system evolves the current at the central $X$ increases $(t=2)$ which, along with the increasing size of the force free region at the primary O's tends to constrict the circulation pattern. In the third frame of Figure $3 a$ we can see that the primary O's have overshot and are now longer top to bottom than side to side. This distortion results in the counter cell seen in the third frame of Figure $3 b$. At about the same time strong negative currents are formed at the ends of the positive current sheet. These drive a back flow which limits the length of the sheet.

\section{Scaling Properties of the Current Sheet}

The morphology of the large-scale flows and current systems are determined by the interaction of the equilibrium field and the perturbation; some of the free energy of the perturbation $(\Delta E)$ is converted into kinetic energy $(k e)$ and some is dissipated in the current sheets $(\Delta m e)$. For our initial conditions $\Delta E \approx$ $\frac{1}{3} T E=9.87$ (where $T E$ is the total energy at $t=0$ ). The morphology and strength of the magnetic fields and flows outside 


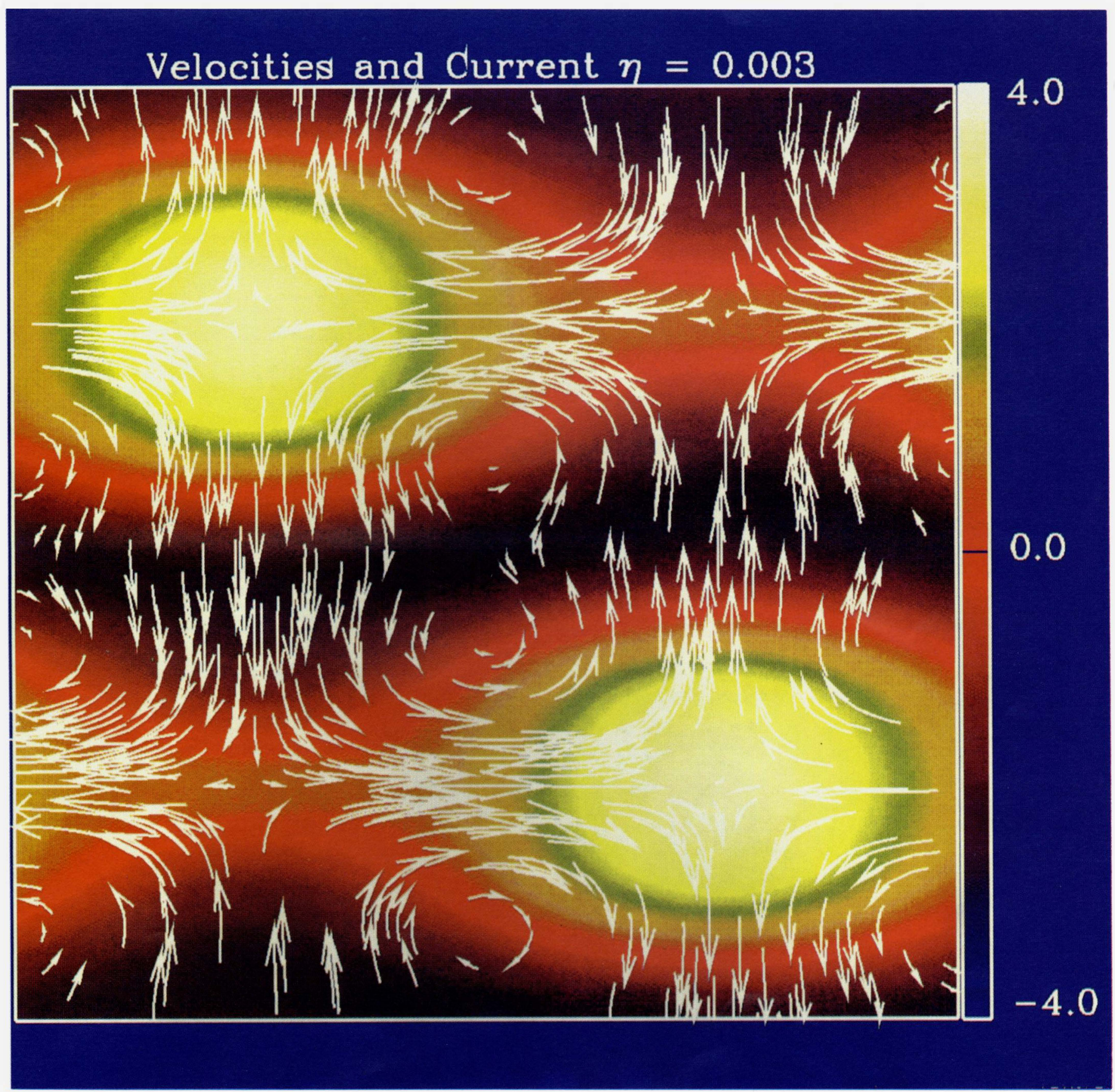

FIG. 2.-The horizontal velocity and vertical current at $t=0.25$

Deluca \& Craig (see 390, 682) 


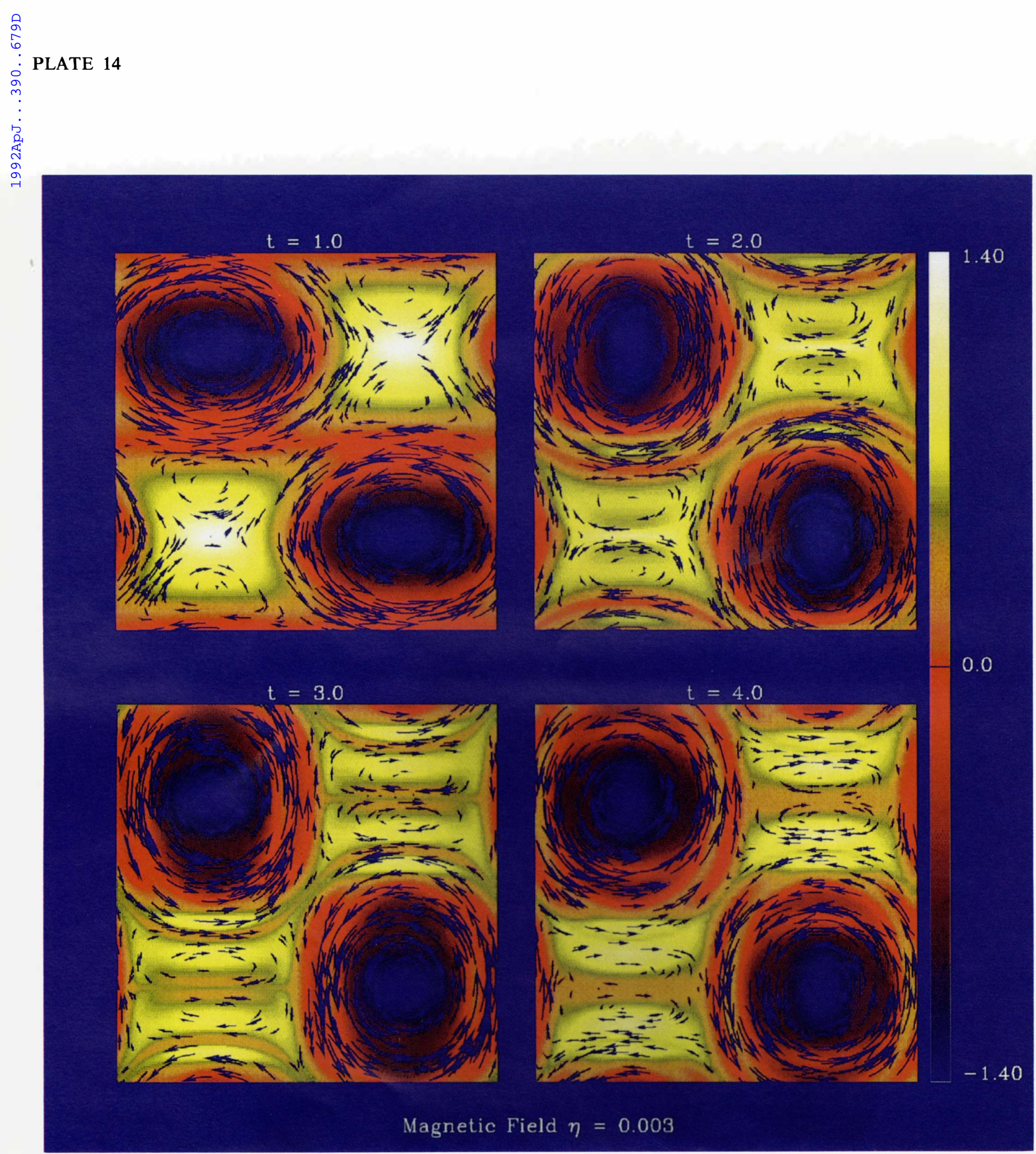

FIG. $3 a$

Fig. 3. $-(a)$ The evolution of the magnetic field; $\eta=0.003$. (b) The evolution of the horizontal velocity and vertical current; $\eta=0.003$.

Deluca \& Craig (see 390, 682) 

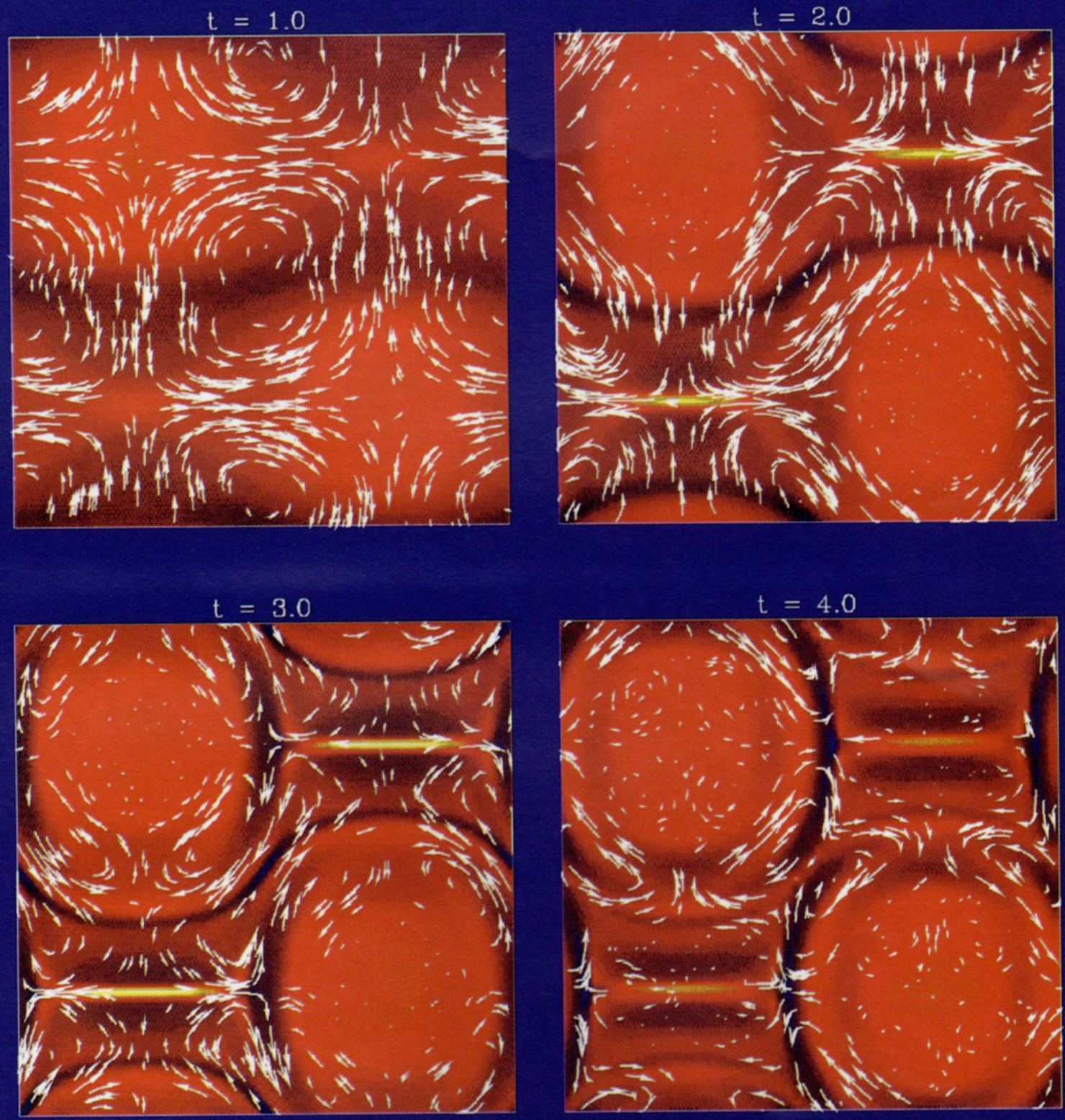

Velocities and Current $\eta=0.003$

FiG. $3 b$

Deluca \& Craig (see 390, 682) 


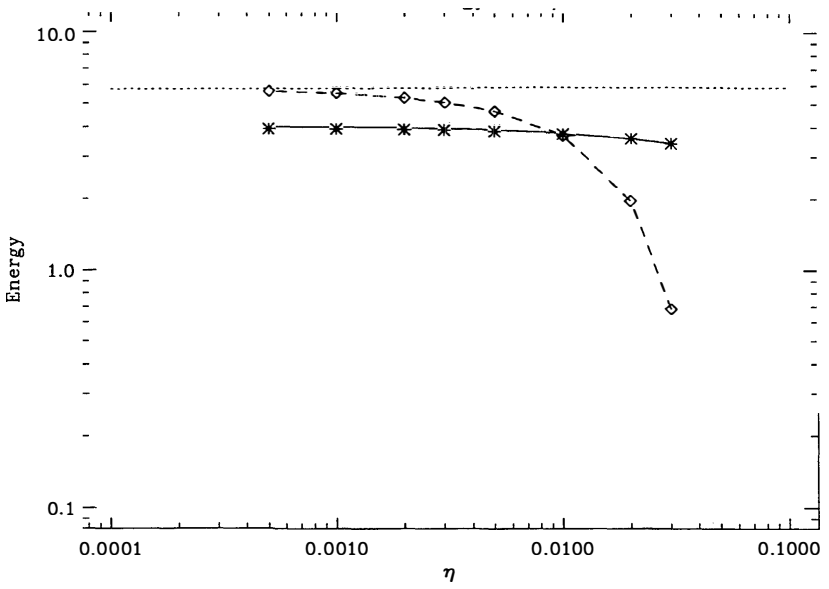

FIG. 4.-Dependence of the free energy on $\eta$. The solid curve shows the maximum kinetic energy as a function of $\eta$. The kinetic energy arises from the magnetic energy of the perturbation, $\Delta E \approx 10$. The dotted line is the diffierence between the asymptotic value of the kinetic energy, $\sim 4$, and the perturbation energy, $\Delta E$. This is value of $\Delta m$ if there was no dissipation. The dashed curve shows the actual value of the free magnetic energy.

the current sheet are unchanged, asymptotically, as $\eta \rightarrow 0$. What does change is the size and strength of the current sheet. One measure of how close we are to the asymptotic limit $\eta \rightarrow 0$ is given by the dependence of $k e$ and $\Delta m e$ on $\eta$. The maximum $k e$ as a function of $\eta$ is shown in Figure 4 (solid curve). (The maximum kinetic energy occurs before the current sheet is formed; $t \approx 1.6$.) It is clear that the maximum $k e$ is approaching a constant value $k e_{0} \sim 4 \approx \frac{2}{5} \Delta E$ as $\eta \rightarrow 0$. (Note that $v=$ $R_{e}^{-1}=10^{-4}<\eta$ for all our simulations). The dotted line shows $\left(\Delta E-k e_{0}\right)$, the value of $\Delta m e$ if there was no dissipation. The dashed curve shows the actual value of $\Delta m e$. The difference between the dotted and dashed curves is a measure of the effect of finite magnetic diffusivity on the large-scale fields (the current sheets have not yet formed). For $\eta<0.01$ we are in the "asymptotic" regime where the fields and flows outside the current sheet are nearly independent of $\eta$. Recall that in $\S 2$ one of the assumptions needed to "derive" the flux pile-up scaling was that $\Delta m e=B^{2} l L \sim \eta^{0}$ (all of the dissipation occurs in the current sheet); Figure 4 shows that this assumption is justified for $\eta<0.01$.

In the remainder of this section we will present an empirical scaling based on our numerical simulations. We take the simplest hypothesis and suppose - in line with the demonstration of Craig \& McClymont (1991) - that the trends shown in the simulations for $\eta<0.01$ will continue to hold as $\eta$ approaches the solar value of $\sim 10^{-12}$. This extrapolation effectively assumes (the tearing mode notwithstanding!) that the current sheet remains stable as $\eta \rightarrow 10^{-12}$.

As previously mentioned, the scaling properties of the length and width of the current sheet together with the current amplitude are sufficient to determine the energy dissipation rate, and the flux annihilation rate. Given that all quantities are strongly time dependent, it is natural to evaluate these parameters at the time of maximum current in the sheet. Evidence from linear studies (see Craig \& McClymont 1991) suggests that fast reconnection at the time of current maximum is indeed a reliable signature of fast reconnection in dynamic studies. Accordingly we shall concentrate on evaluating the energy dissipation rate at the time of the current maximum.

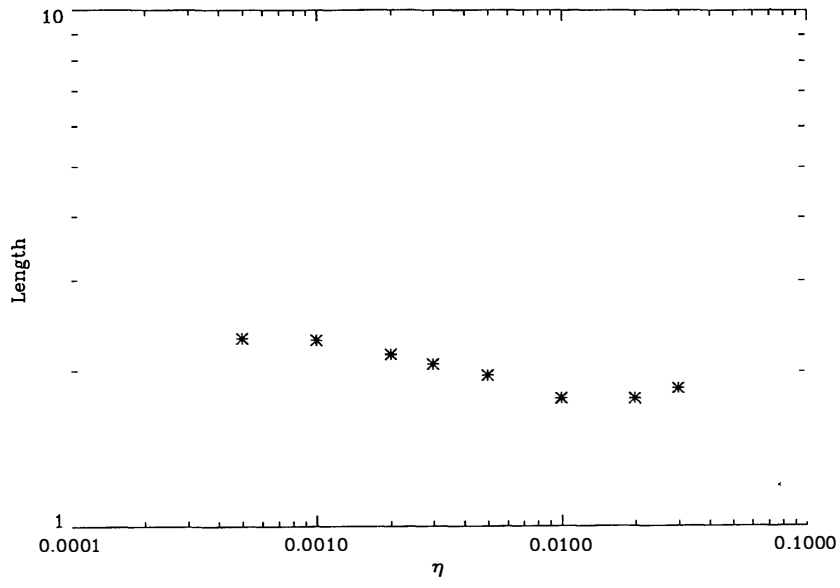

Fig. 5.-Dependence of the current sheet length, $L$, on $\eta$. $L$ becomes independent of $\eta$ as $\eta \rightarrow 0$.

Figure 5 shows the length of the current sheet as a function of $\eta$. The length is defined as the distance between the peaks in the outflow velocity a long the current sheet. (The same definition as Biskamp 1986 used in his numerical solutions.) The length is seen to be approaching a fixed value of $\sim 2.3$ for small $\eta$. That the maximum current sheet length is determined ultimately by the geometry, is an almost universal result of finite amplitude simulation studies (see Biskamp 1986; Biskamp \& Welter 1989; Strauss 1990).

Figure 6 shows the power-law behavior of the width, $l$. We again follow Biskamp (1986) and define the width of the current sheet by fitting the current profile with the function: $J=J_{\max } /\left(\cosh \left[\left(x-x_{0}\right) / l\right)\right]^{2}$, where $x_{0}$ is the location of the maximum current. Two fiducial lines are drawn with slopes of 0.5 (solid line) and 0.6 (dashed line). The product of the length and the width is shown in Figure 7. The area of the current sheet appears to scale as $\eta^{1 / 2}$, consistent with the flux pile-up model.

Finally Figure 8 shows the power-law behavior of the maximum current. Again we provide reference lines for the points $\eta<=0.01$; where Figure 4 suggests our model should be valid. Comparison of the points with the two lines shows that the current seems to be turning over at the smallest values

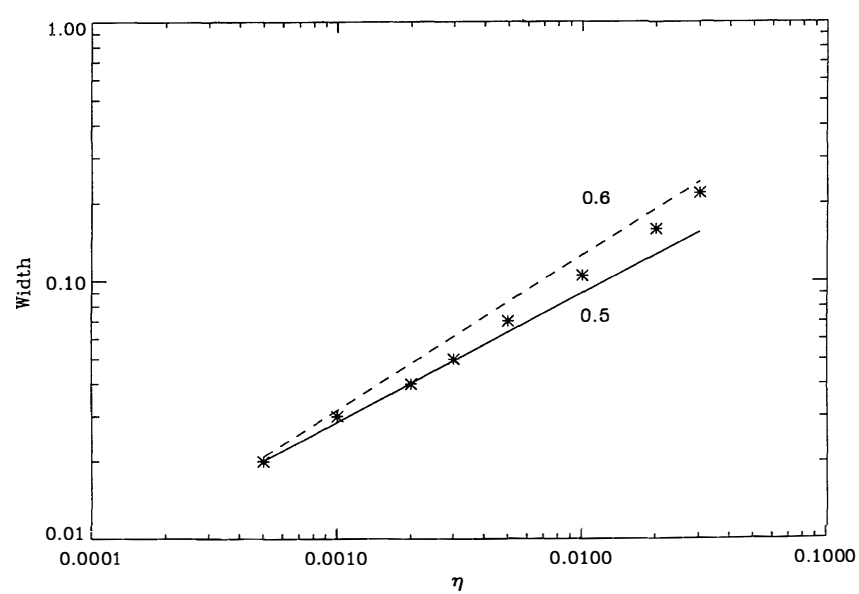

FIG. 6.-Scaling of the current sheet width. Fiducial lines with scaling of $\eta^{0.5}$ and $\eta^{0.6}$ are presented for reference. 


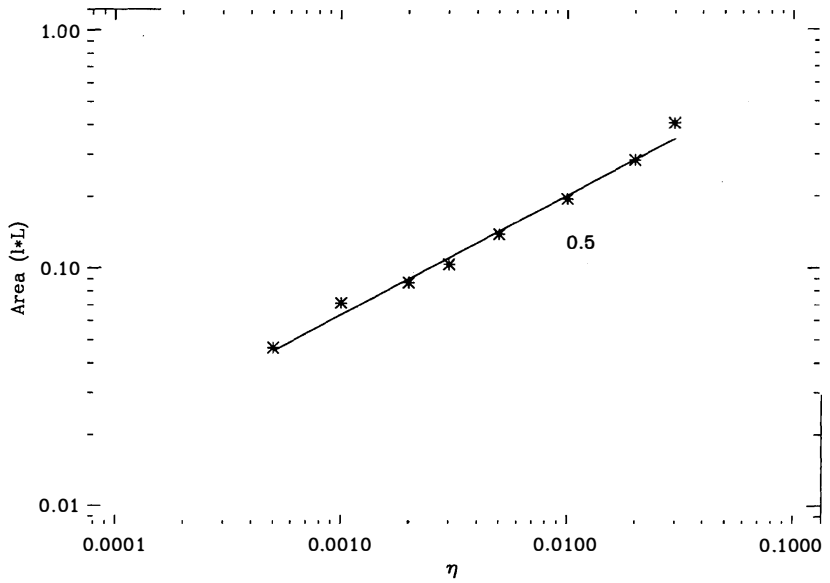

FIG. 7.-Scaling of the current sheet area. A line with slope 0.5 is shown for reference.

of $\eta$. It is not clear whether this is an indication of the flux pile-up stalling (as suggested by Biskamp \& Welter 1980) or is due to inadequate resolution for the final data point. The previous points are well fitted by an $\eta^{-3 / 4}$ power law.

In Figure 9 we show scaling of the dissipation rate $W_{\eta}$. The solid line is $W_{n} \sim \eta l L J_{\max }^{2}$, and the dashed line is $W_{n}=$ $\sum \eta J^{2} \delta x \delta y$ over the current sheet. The latter calculation is somewhat noisy because the current sheet is very thin, but both curves show the tendency for the dissipation rate to level off at small values of $\eta$. (For marginally resolved computations the dissipation rate will be underestimated - to this extent our results give a lower limit to both current maximum, and the reconnection rate.)

Another way of checking the flux pile-up hypothesis is to consider the scaled current $\eta J^{4 / 3}$. This quantity is independent of $\eta$ in the flux pile-up model. Figure 10 shows the time evolution of the scaled current for $(\eta \leq 0.005)$ for 3 Alfvén transite times. A weak dependence on $\eta$ is seen in the mild decline of the reconnection rate compared with the theoretical prediction. We are unsure at this moment whether this behavior is just an artifact of the decreased (relative) resolution at low $\eta$, or whether a breakdown of the flux-limited hypothesis is signalled at small enough resistivities. Figure 10 also shows that the time

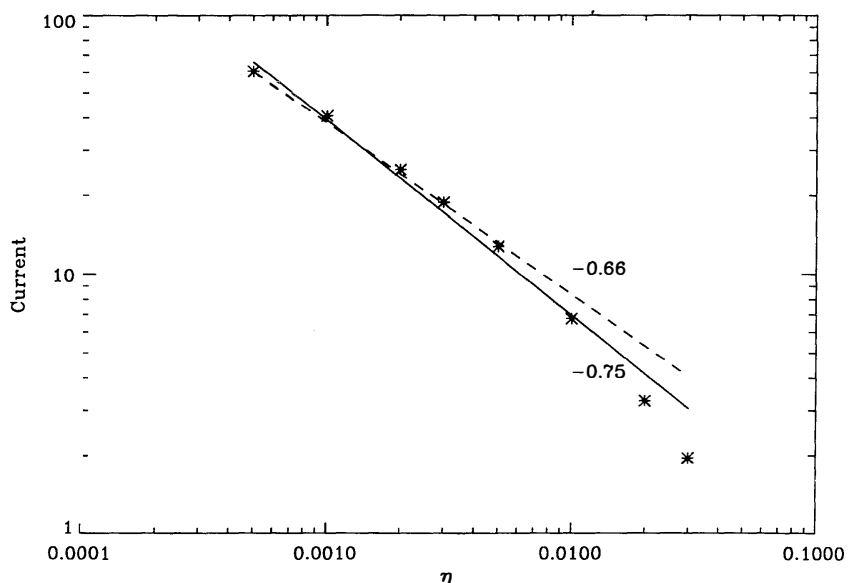

FIG. 8.-Scaling of the maximum current. Fiducial lines with scaling of $\eta^{-0.75}$ and $\eta^{-0.66}$ are presented for reference.

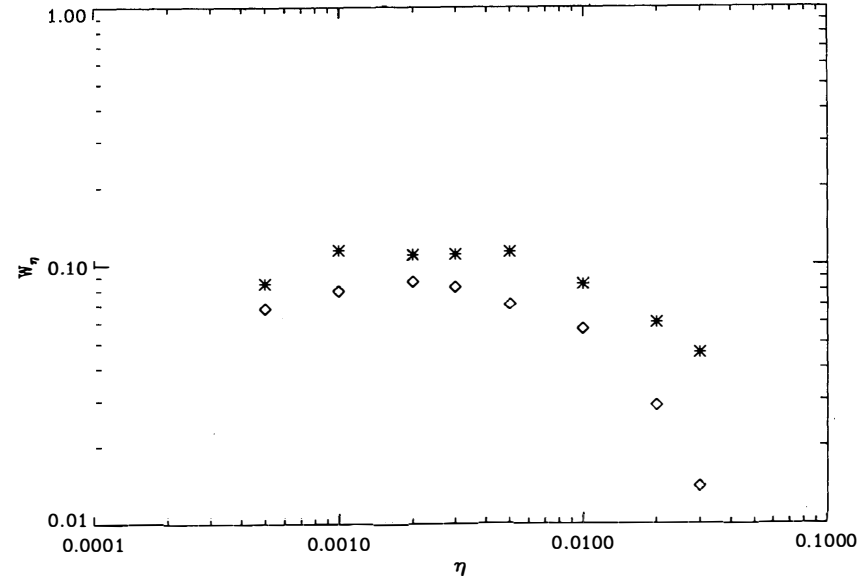

FIG. 9.- The energy dissipation rate, $W_{\eta}$. The stars show $W_{\eta}$ as determined from the measurements of the length, width and maximum current. The squares show $W_{\eta}$ based on the sum of $\eta J^{2}$ over the current sheet in the solutions. The former curve lies above the latter as $J_{\max }^{2} \geq J^{2}$.

of maximum current is a function of $\eta$, this means that even if the dissipation rate is independent of $\eta$ the reconnection time may have a weak time dependence due to the dependence of the collapse time on $\eta$ (Vainshte in 1991). We are in the process of investigating further the dependence of the collapse time on $\eta$.

We have examined the other scaling properties of the solutions around the current sheet and have found them to be consistent with our model of a flux pile-up solution. For example, both the maximum amplitude of the magnetic field parallel to the current sheets $\left(B_{x}\right)$ and the integrated current scale should scale as $\eta^{-0.25}$ in the flux pile-up model. The small $\eta$ scalings for these two quantities are $\sim 0.2$ and 0.3 , respectively.

\section{CONCLUSIONS}

\subsection{Summary and Future Work}

We have demonstrated that within the present parameter range, the flux pile-up model of fast reconnection provides the

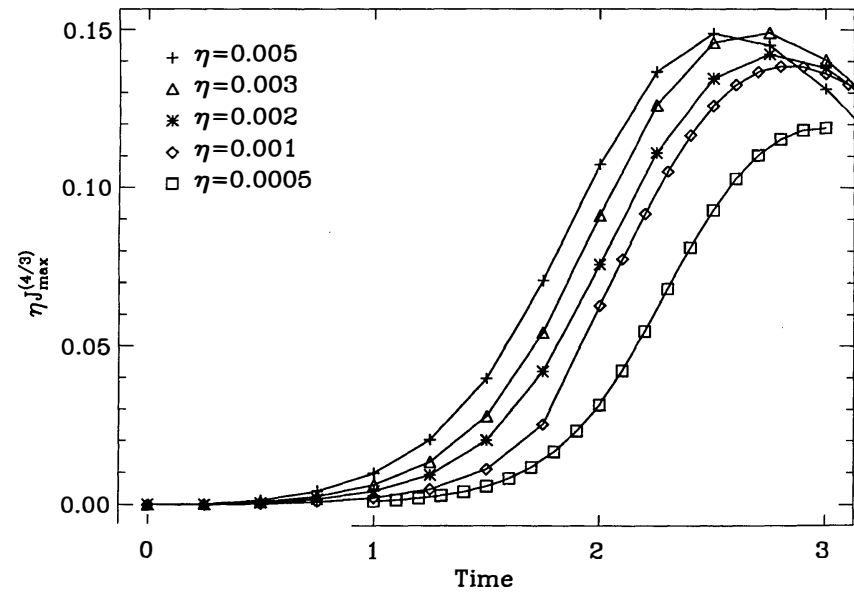

Fig. 10.-Time evolution of the scaled current $\eta J^{4 / 3}$, for five different values of $\eta$. The flux pile-up hypothesis predicts that $\eta J^{4 / 3}$ is independent of $\eta$. We see a weak dependence here. Note also that the time of current maximum is dependent on $\eta$. 
most convincing explanation of our results. The energy dissipation occurs via the formation of classical X-type neutral points in a divergent flow topology (the flow being directed away from, rather than into, current sheets; see Forbes \& Priest 1987). The reconnection rate remains fast because, in a dynamic calculation, reductions in the plasma resistivity are compensated by enhanced magnetic field intensities-i.e., the pile-up of flux - at the edge of the current sheet. Craig \& McClymont (1992) also see flux pile-up reconnection in their two-dimensional, compressible (no gas pressure), finite amplitude calculations. However, their fast reconnection stalls when sufficient gas pressure is introduced into the problem. When there is no gas pressure the sound speed is zero and there is no hindrance to flux-pile up in simple single X-type neutral point. However, for finite sound speed (or infinite sound speed as in the present calculations) a more complex flow topology is needed to prevent the reconnection from stalling. The present calculation shows that finite sound speed-and finite perpendicular field-need not inhibit fast reconnection in more general flow topologies.

Interestingly, Strauss (1990) argues for fast reconnection in his three-dimensional driven problem. He also sees evidence for flux pile-up with scalings comparable to the Biskamp \& Welter model of Table 1. This result is somewhat enigmatic, however, since Biskamp \& Walter assert the breakdown of these scalings at small enough resistivity. That fast reconnection may stall at low enough resistivity is certainly a theoretical possibility which cannot be overruled - and may even be suggested at the lower end of our resistivity range-by the present computations. None the less the dominant trends suggested by our best-resolved computations seem remarkably consistent with the flux pile-up fast reconnection hypothesis. If these results are confirmed with independent high-resolution calculations, then we can expect fast reconnection to be a common feature of astrophysical plasmas.

On a practical level it is clear that higher resolution runs for smaller values of $\eta$ are needed to firm up the physical picture. Some of these will be done with the existing spectral code. In addition we are working on a new spectral code which will allow us to use Chebyshev polynomials with the end points located at the current sheets. This will concentrate the collocation points in the current sheet where high resolution is essential.

\subsection{Implications for the Solar Atmosphere}

The flux pile-up model is attractive for solar flares because the free energy of the perturbed magnetic field is converted directly into heat. In contrast, the Petschek (1964) model and the linear Craig \& McClymont (1991) model convert the free energy into kinetic energy of strong outflows along the sheet which must then be transformed into heat by some other mechanism. The flux pile-up model is also distinguished by its large aspect ratio $(L / l)$, compared wth the Petschek and Craig \& McClymont models.

We examine, briefly, the possibility that there are observational implications of our scaling law. Our current sheets are characterized by their large aspect ratio, and a rise time of a few Alfvén times. If there were no conductive cooling we might expect high spatial and temporal resolution X-ray observations, from say the SXT on Solar-A, to see thin lines of emission brightening nearly uniformly before the loop is filled with evaporated material from the chromosphere. Unfortunately, estimates of the emission measure based on our incompressible
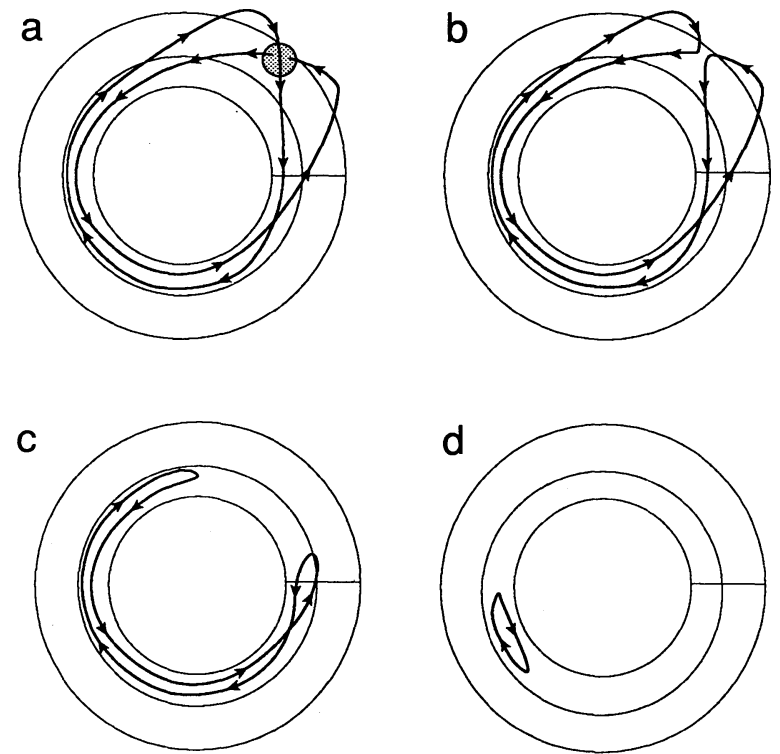

FIG. 11. $-(a-d)$ The change in topology of two toroidal fibrils due to reconnection in the shaded area. The fibril fields are projected onto the equatorial plane. Both fibrils are anchored in the overshoot layer and extend up through the convection zone and the photosphere. After the reconnection takes place the single fibril can contract, and dissipate as an isolated ring of field. Notice that the net unsigned flux through the plane is reduced as a result of the reconnection.

calculation indicate that the initial brightening would be well below the threshold of detectability for the Solar-A. Density enhancements (the result of a compressible flow) during the collapse of the current sheet are probably needed for the early brightening of the current sheet to be detectable.

\subsection{Implications for the Solar Cycle}

Fast reconnection of fibril fields within the solar convection zone can provide a way of changing the sign of the toroidal field every $11 \mathrm{yr}$ without resorting to turbulent diffusion (Parker 1982a, b). A simple cartoon model is presented in Figure 11. Here we have projected the northern hemisphere convection zone onto the equatorial plane. We consider two oppositely directed flux tubes, rooted in the overshoot region. The unsigned flux across a meridional plane (indicated by the radial line in the figure) is $2 \Phi$, where $\Phi$ is the strength of each flux tube. If $\eta=0$ the unsigned flux cannot decrease below 2 (unless the fibrils slip over the top of the pole). However, if convective motions bring the tubes close together at one point, and the tubes reconnect to form a single tube, then the unsigned flux can vanish. Once the topology is changed the fibril can collapse on itself and the flux will dissipate into heat.

We expect reconnection to occur when the time scale for reconnection is short compared with the dynamical time scale that brings the fibrils together and pulls them apart. Our calculations show that the reconnection time scale is several, say 20 , Alfvén transit times. Consider a flux tube with $B=10^{4} \mathrm{G}$, and $\Phi=10^{21} \mathrm{Mx}$, near the base of the convection zone, where $\rho=0.2 \mathrm{~g} \mathrm{~cm}^{-3}$. The Alfvén speed is $V_{A} \approx 6 \times 10^{3} \mathrm{~cm}^{-1}$, while the diameter of the tube is $L=3 \times 10^{8}$. This leads to a time scale of $t_{r} \sim 20 L / V_{A}=10^{6} \mathrm{~s}$ or 12 days. The turnover time in the convection zone is around 26 days. Clearly, there is no difficulty reconnecting two fibrils brought together by motions deep in the convection zone. 
Consider for a moment fibrils with a fixed amount of flux $\Phi$ as the fibril stretched both the Alfvén speed will increase and the tube diameter will decrease. The time scale for reconnection is proportional to the $-3 / 2$ power of $B$. So a $10^{5} \mathrm{G}$ tube with $10^{21} \mathrm{Mx}$ will reconnect in less than a day (assuming a 20 Aflvén transit time reconnection rate). Fibrils, subject to aerodynamic drag, are able to be stretched until the field strength is well above the equipartition strength (Parker 1982a, b). The above reconnection rates indicate that the topology of these strong fibrils can change very quickly.

Vainshtein, Parker, \& Rosner (1991) have reformulated the mean field dynamo equations to include the effiects of reconnection on the diffusion of strong toroidal fields. They have made the conservative assumption that reconnection proceeds at the Sweet-Parker rate $\eta^{1 / 2}$. Our results suggest that the reconnection may proceed at a much faster rate which is logarithmic in the plasma resistivity.

The authors have benefited from discussions with many individuals during the course of this work. We would particularly like to thank F. Cattaneo, G. Fisher, A. McClymont, E. Parker, S. Vainshtein, and R. Rosner for their many insightful comments. This work is supported by Air Force grant AFOSR-90-0116, NASA grant NAGW-864, and NSF grant ATM-9106052.
Biskamp, D. 1986, Phys. Fluids, 29, 1520

Biskamp, D., \& Welter, H. 1980, Phys. Rev. Lett., 44, 1069 1989, Phys. Fluids, 1, 1964

Canuto, C., Hussaini, M. Y., Quarteroni, A., \& Zang, T. A. 1988, Spectral Methods in Fluid Dynamics (New York: Springer)

Cattaneo, F., \& Vainshtein, S. I. 1991, ApJ, 376, L21

Craig, I. J. D., \& McClymont, A. N. 1991, ApJ, 371, L41 1992, in preparation

Craig, I. J. D. , \& Watson, P. W. 1991, ApJ, in press

DeLuca, E. E., \& Gilman, P. A. 1991, in The Solar Atmosphere and Interior, ed. A. Cox, (Tucson: Univ. of Arizona Press)

Forbes, T. G., \& Priest, E. R. 1987, Rev. Geophys., 35, 1583

Parker, E. N. 1957, J. Geophys. Res., 79, 1558

. 1963, ApJS, 77, 8
REFERENCES

Parker, E. N. 1979, Cosmical Magnetic Fields (Oxfiord Univ. Press) 1982a, ApJ, 256, 292 1982 b, ApJ, 256, 302

Petschek, H. E. 1964, in AAS-NASA Symposium on Physics of Solar Flares (NASA SP-50), ed. W. N. Hess (Washinton DC: NASA)

Spitzer, L. 1956, The Physics of Fully Ionized Gases (New York: WileyInterscience)

Stix, M. 1989, The Sun: An Introduction (Berlin: Springer)

Strauss, H. R. 1990, J. Geophys. Res., 95, 17145

Sweet, P. A. 1958, in IAU Symp. 6, Electromagnetic Phenomena in Cosmica Physics, ed. B. Lehnert (Cambridge Univ. Press), 123

Vainshtein, S. I. 1991, private communication

Vainshtein, S. I., Parker, E. N., \& Rosner, R. 1992, ApJ, submitted

Vainshtein, S. I., \& Rosner, R. 1991, ApJ, 376, 199 